3-1-2016

\title{
Fabrication and Experimental Analysis of Axially Oriented Nanofibers
}

Ashish Aphale

Kapil Mahakalkar

Isaac Macwan

Ishita Mukerji

Paris J. Cox

See next page for additional authors

Follow this and additional works at: https://digitalcommons.fairfield.edu/engineering-facultypubs Copyright (C) 2015 American Scientific Publishers. All rights reserved.

Publisher PDF has been archived here with permission from the copyright holder.

\section{Peer Reviewed}

\section{Published Citation}

Aphale, A. N., Mahakalkar, K., Macwan, I. G., Mukerji, I., Cox, P. J., Mahapatra, M., Singh, P., Ajayan, P.M., Patra, P. K. (2016). Fabrication and Experimental Analysis of Axially Oriented Nanofibers. Journal of Nanoscience and Nanotechnology, 16(3), 2668-2676. https://doi.org/10.1166/jnn.2016.10827

This item has been accepted for inclusion in DigitalCommons@Fairfield by an authorized administrator of DigitalCommons@Fairfield. It is brought to you by DigitalCommons@Fairfield with permission from the rightsholder(s) and is protected by copyright and/or related rights. You are free to use this item in any way that is permitted by the copyright and related rights legislation that applies to your use. For other uses, you need to obtain permission from the rights-holder(s) directly, unless additional rights are indicated by a Creative Commons license in the record and/or on the work itself. For more information, please contact digitalcommons@fairfield.edu. 


\section{Authors}

Ashish Aphale, Kapil Mahakalkar, Isaac Macwan, Ishita Mukerji, Paris J. Cox, Manoj Mahapatra, Prabhakar Singh, Pulickel M. Ajayan, and Prabir K. Patra 


\title{
Fabrication and Experimental Analysis of Axially Oriented Nanofibers
}

\author{
Ashish N. Aphale ${ }^{1}$, Kapil Mahakalkar², Isaac G. Macwan ${ }^{1}$, Ishita Mukerji ${ }^{3}$, Paris J. Cox ${ }^{4, \dagger}$, \\ Manoj Mahapatra ${ }^{5}$, Prabhakar Singh ${ }^{5}$, Pulickel M. Ajayan ${ }^{6}$, and Prabir K. Patra ${ }^{2,7, *}$ \\ ${ }^{1}$ Department of Computer Science and Engineering, University of Bridgeport, Bridgeport, CT 06604, USA \\ ${ }^{2}$ Department of Biomedical Engineering, University of Bridgeport, Bridgeport, CT 06604, USA \\ ${ }^{3}$ Department of Molecular Biology and Biochemistry, Molecular Biophysics Program, Wesleyan University, \\ Middletown, CT 06457, USA \\ ${ }^{4}$ Department of Mechanical Engineering, Rice University, Houston, Texas, 77005, USA \\ ${ }^{5}$ Department of Materials Science and Engineering, University of Connecticut, Storrs, CT, 0629, USA \\ ${ }^{6}$ Department of Materials Science and Nanoengineering, Rice University, Houston, Texas 77005, USA \\ ${ }^{7}$ Department of Mechanical Engineering, University of Bridgeport, Bridgeport, CT 06604, USA
}

\begin{abstract}
A novel design of a laboratory built axially rotating collector (ARC) having capability to align electrospun nanofibers have been described. A detailed morphological comparison of such nanofibers orientation and their geometry is done using scanning electron microscopy (SEM). For comparison various polymeric solutions were electrospun on conventional static collector as well as ARC. The average diameter of polyvinyl alcohol (PVA) nanofibers was found to be $250 \mathrm{~nm}$ while polycaprolactone $(\mathrm{PCL})$ nanofibers were found to be within a range of $600-800 \mathrm{~nm}$. Conducting nanoparticles such as graphene and multi-walled carbon nanotubes (MWNTs) mixed with polymer solutions shown to have a significant influence on the overall geometry of these nanofibers and their diameter distribution. It is evident from the SEM analysis that both graphene and MWNTs in polymer solution play a crucial role in achieving a uniform diameter of nanofibers. Lastly, the formation of the aligned nanofibers using ARC has been mathematically modeled and the electromagnetic field governing the process has been simulated.
\end{abstract}

Keywords: Polyvinyl Alcohol, Polycaprolactone, Graphene, MWCNT, Electrospinning.

\section{INTRODUCTION}

Electrospinning is a versatile tool to fabricate nanofibers from various materials such as biopolymers, engineering plastics, conductive polymers, polymer blends and ceramics with diameters ranging from micro to nanometer scale. ${ }^{1-4}$ The healthcare profession has employed biocompatible materials with particular properties such as mechanical strength, flexibility, moisture and air permeability for medical and surgical applications. ${ }^{5}$ Electrospun nanofibers also have a wide range of applications in composite reinforcement, membrane based separation, and tissue engineering. ${ }^{6}$ Polymer nanofibers have also

\footnotetext{
*Author to whom correspondence should be addressed.

Present Address: Department of Mechanical Engineering, Massachusetts Institute of Technology, Cambridge, MA 02139, USA.
}

been fabricated with a wide range of properties, such as electrical conductivity, low weight, and low porosity along with tailored surface functionalities. ${ }^{7,8}$ Previous work has been done to produce nanofibers containing nanoparticles such as carbon nanotubes $(\mathrm{CNTs})^{9}$ and graphene. ${ }^{10}$ Graphene and CNTs are atomically thin materials made up of carbon atoms having tremendous electrical, thermal and mechanical properties. ${ }^{11-13}$ There is a great interest in developing nanostructured materials using graphene and CNTs with polymer nanocomposites for a variety of applications such as energy storage, ${ }^{14}$ filtration membrane, ${ }^{15}$ scaffolds to grow artificial tissues ${ }^{16}$ and the like.

Nano and micro fibers are specifically important in biomedical engineering as they mimic the scales of natural cellular environments. ${ }^{17,18}$ In general, fibers usually have a random orientation and are collected as a random 
mat with a minimal control on their structural orientations. It has been shown previously by Reneker and co-workers that fiber alignment can be altered by modifying the collector geometry. ${ }^{19}$ For instance, a tubular scaffold with obliquely aligned fibers was constructed on a rotating rod between electrodes. ${ }^{20}$ Several other techniques have also been proposed to produce aligned nanofibers such as, but not limited to, rotating drum collector design, ${ }^{3,21}$ an auxiliary electrode and electrical field technique, ${ }^{22,23}$ a spinning wheel with a sharp edge technique, ${ }^{24}$ a frame collector technique, ${ }^{25}$ a multiple field technique ${ }^{26}$ and a dual vertical wire technique. ${ }^{27}$ We report the design of a new collector geometry called Axially Rotating Collector (ARC) that can align the deposited nanofibers coaxially on the rotating disc (collector). To test the collector geometry and to compare the resulting hybrid nanofibers, poly (vinyl alcohol) (PVA), composite PVA-MWNTs polymer solution, Polycaprolactone (PCL) and PCL-graphene were used.

\section{MATERIALS AND METHODS}

Rotating collector geometry was designed with the capability to produce aligned nanofibers. The collector geometry design consisted of an aluminum disc mounted on the shaft of a mechanical motor running at a constant speed of $1700 \mathrm{rpm}$ as shown in Figure 1(A). The ground wire is connected behind the collector and a class 1 copper switch module is used for a permanent ground connection with the plate even when it is rotating at a high speed
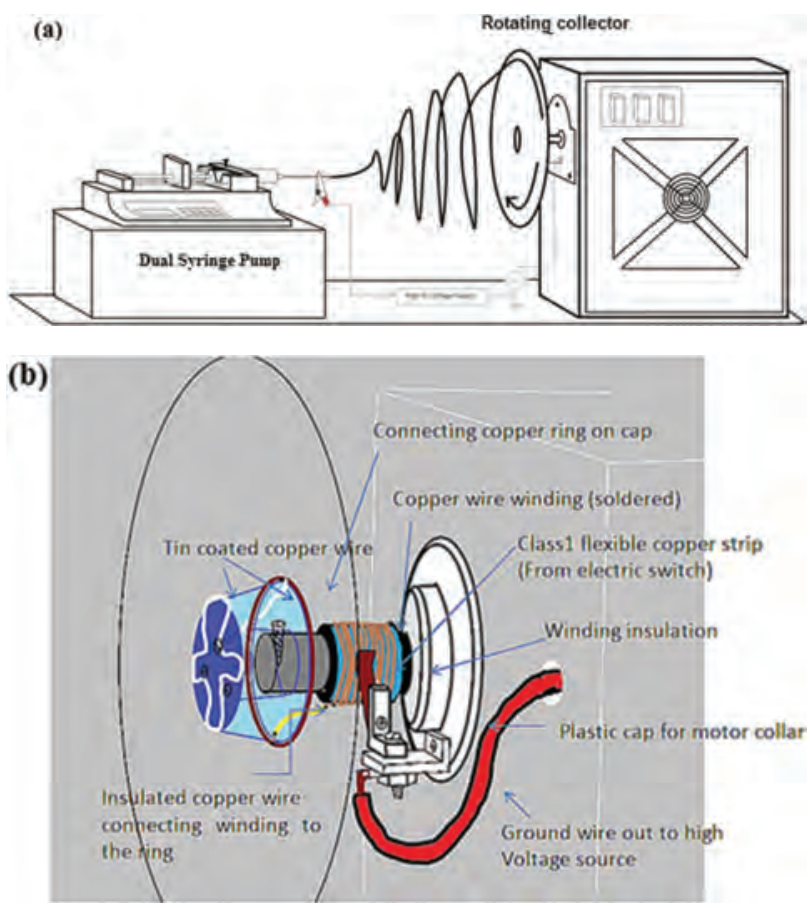

Figure 1. (a) Schematic illustration of ARC collector geometry having capability to align nanofibers, (b) detailed description of the grounding mechanism of collector. as shown in Figure 1(B). The collector thus rotates at about $1700 \mathrm{rpm}$ in clockwise direction and the nanofibers are deposited coaxially on the surface of the aluminum disc.

To test the collector geometry and its effect on the overall nanofiber characteristics such as nanofibers diameter and orientation, several experiments were conducted using polycaprolactone (PCL), polyvinyl alcohol (PVA) and their composite solutions. To see the effect of conductive nanoparticles on the fiber geometry and orientation, graphene and multi-walled carbon nanotubes (MWNTs) were used along with PCL and PVA solutions respectively. The electrospinning setup comprised of a dual syringe pump (KDS 200 series, KDS scientific), a high voltage power supply (Model ES30P, Gamma High Voltage Research) and a metallic (aluminum) collector placed at an appropriate distance from the tip of the needle. The variables that are important in electrospinning of nanofibers are, the distance between the needle and the collector $(d)$, viscosity of the polymer solution, flow rate $(r)$ of the polymer solution from the needle tip through the syringe and electric field strength applied which is voltage per distance $(\mathrm{kV} / \mathrm{cm})$ between the needle and the collector.

$10 \mathrm{wt} \%$ PCL solution (Sigma Aldrich, 70 k-90 k Mw) was prepared using $10 \mathrm{~g}$ of PCL pallets in $100 \mathrm{ml}$ acetone solvent and stirring at $60{ }^{\circ} \mathrm{C}$ until a clear solution was achieved. Polymeric nanocomposite solution was also prepared by adding different concentrations of graphene in $10 \mathrm{wt} \%$ PCL solutions. A weighted quantity of graphene (Cheaptubes Inc.) was used to prepare $0.01 \mathrm{wt} \%$ and $0.005 \mathrm{wt} \%$ concentration and dispersed in acetone by sonicating for $1 \mathrm{hr}$ under no heat followed by required quantity of PCL to make $10 \mathrm{wt} \%$ concentration of PCL-graphene solution. PVA solution was prepared in deionized water with a concentration of $10 \mathrm{wt} \%$ by measuring a weighted amount of PVA powder (10 g) (Sigma Aldrich, $61 \mathrm{k} \mathrm{Mw}$ ) and dissolving it in $100 \mathrm{ml}$ water at $80{ }^{\circ} \mathrm{C}$ followed by stirring the solution for 2 hours until a sufficient solution of PVA was achieved. PVA- MWNT solution was prepared by adding $10 \mathrm{mg}$ MWNTs in $10 \mathrm{ml}$ of $10 \mathrm{wt} \%$ PVA solution. The mixture was stirred for one hour and further dispersion was achieved by sonicating for another hour. Control PCL and PCL-graphene composite nanofibers were electrospun by applying a fixed DC potential of $17 \mathrm{kV}$ across a distance of $15 \mathrm{~cm}$ at a flow rate of $1.5 \mathrm{ml} \mathrm{hr}^{-1}$. Electrospinning of PVA solution was performed at $15 \mathrm{kV}$ across $15 \mathrm{~cm}$ distance at a flow rate of $1.5 \mathrm{ml} \mathrm{hr}^{-1}$. These conditions were optimized after several experiments. Scanning electron microscope (SEM) was utilized to characterize the morphology of the fibers after sputter coating (CRC 50 Sputter Coater) gold on the sample for $25 \mathrm{~s}$. The SEM images were analyzed using image processing software (ImageJ) to determine the average fiber diameter. Distribution of fiber diameter was calculated from 20 random measurements per image. 


\section{RESULTS AND DISCUSSION}

As shown in Figure 2 it can be clearly observed that the ARC collector has capabilities to orient the nanofibers. Further it can be observed that the addition of conductive nanomaterial such as graphene or MWNTs have influenced the overall nanofibers size distribution and their geometry. SEM images revealed that the control $10 \%$ PCL nanofibers regardless of their orientation have mean fiber diameter in the range of 600-800 $\mathrm{nm}$. The nanofibers from 10\% PCL solution fabricated using ARC collector can produce majority of fibers aligned in one direction however they tend to have a wavy pattern. This wavy pattern may have occurred because of the collector which rotates at a high speed creating miniscule vibrations and other mechanical disturbances. It is further observed that the addition of graphene increases the consistency of the nanofiber diameter when compared to the case where there is no graphene involved. In the case of $10 \%$ PCL-0.005 wt $\%$ graphene (aligned) the average fiber diameter is approximately $900 \mathrm{~nm}$. Interestingly the wavy pattern of the nanofibers which was more prominent in the case of control 10\% PCL reduced significantly. It is also interesting to observe that upon the inclusion of graphene in PCL solution, several individual nanofibers tend to coalesce. Increasing the concentration of graphene, $10 \%$ PCL-0.01 wt\% graphene, caused a significant change in overall fiber geometry as diameter of the nanofiber is observed to be consistent across its length. A relatively "tight" distribution of the aligned nanofiber diameter is observed compared to others. Average fiber diameter is in the range of 600-800 $\mathrm{nm}$ for aligned nanofibers compared to $700-900 \mathrm{~nm}$ for randomly oriented nanofibers. Improvement in the fiber orientation and overall consistent fiber diameter across its length can be attributed to the inclusion of conductive nanomaterial graphene. Graphene creates a localized conductive spot which helps electrical charges to concentrate on the beads that act as electrical stress centers which possibly lead to continuous stretching of the fibers. However, thorough investigation is needed to strengthen this hypothesis.

Similarly, controlled PVA solution with $10 \mathrm{wt} \%$ and 15 wt $\%$ concentration were electrospun to observe the effect of PVA concentration of the nanofibers collected via the ARC collector. To understand the effect of conductive nanoparticle, multi-walled nanotubes (MWNTs) with concentration of $0.1 \mathrm{wt} \%$ in $10 \mathrm{wt} \%$ PVA solution were electrospun. As shown in Figures 3(A), (B) it is observed that randomly oriented nanofibers from $10 \mathrm{wt} \%$ PVA solution have an average diameter in range of $80 \mathrm{~nm}$ to $170 \mathrm{~nm}$ while $15 \mathrm{wt} \%$ PVA solution tends to have higher fiber diameter in the range of $100 \mathrm{~nm}$ to $270 \mathrm{~nm}$ as shown in Figures 3(C), (D). The increase in overall diameter of nanofibers with the increase in concentration of PVA solution can be attributed to the increase in viscosity of PVA, which leads to less stretching of each single nanofiber while being electrospun. Interestingly, the addition of $0.1 \mathrm{wt} \%$ MWNTs in the $10 \mathrm{wt} \%$ PVA solution significantly altered the structural morphology of electrospun nanofibers. The PVA-MWNT nanofibers appeared as physically entangled networks, with the average diameter of $250 \mathrm{~nm}$. It was also observed that the fibers produced from the composite solution have a continuously uniform diameter along the length with no bead formation (Figs. 3(E), (F)). Electropsining of 10\% PVA control solution using ARC collector also fabricated majority of aligned nanofibers as shown in Figure 4. The average diameter of aligned nanofibers is $250 \mathrm{~nm}$. Several individual single nanofibers are bundled together making thicker threads that run parallel to each other exhibiting a stronger fiber structure. There are few single nanofibers that are randomly oriented, which may be due to the vibration of the rotating plate or air turbulence.

A drop of polymeric solution from the syringe needle starts to stretch when an electric potential of $15 \mathrm{kV}$ is applied. On the other hand, the surface tension of the polymer solution holds the drop back and in the process the polymer drop gets stretched to form a fiber. When a single fiber from the drop reaches the ARC which is rotating at $1700 \mathrm{rpm}$, the nanofibers come under a tremendous tensile force and get elongated even further. This leads to the nanofibers placed along the direction of the rotation on the collector geometry and this process is repeated for each and every fiber that comes out of the drop from needle tip. Hence, they get deposited in a concentric fashion and are aligned in the direction of the rotation perpendicular to the axis of the rotation of ARC.

We created a computer model for the syringe and the disc collector in order to understand the electric field patterns that are generated in the surrounding medium (air) between the needle and the collector during the electrospinning of the nanofibers. Figure 5 shows the electric field in the form of an isosurface, where we can clearly see the electric field lines targeting the grounded aluminum collector at its periphery. The needle is modeled as a high-strength alloy steel cylinder with $40 \mathrm{~mm}$ length and $0.8 \mathrm{~mm}$ diameter. Similarly, the aluminum disc collector is modeled as a $300 \mathrm{~mm}$ diameter disc having a thickness of $1 \mathrm{~mm}$. Material properties are assigned as per standard values for relative permittivity and electric conductivity for the needle, disc and the surrounding air. It is known that air is a very good insulator, however, at high voltages such as in case of electrospinning $(\sim 15 \mathrm{kV})$, the medium between the needle and the collector ionizes thereby slightly increasing the conductivity and hence to accommodate this aspect, an initial electric conductivity of $1 \times 10^{-14} \mathrm{~S} / \mathrm{m}$ for air is considered. Furthermore, a tetrahedral meshing algorithm was used for finite element analysis and 3D as well as 2D plot groups in COMSOL multiphysics were utilized for the analysis of the resulting electric field. It was found that the aluminum collector is 

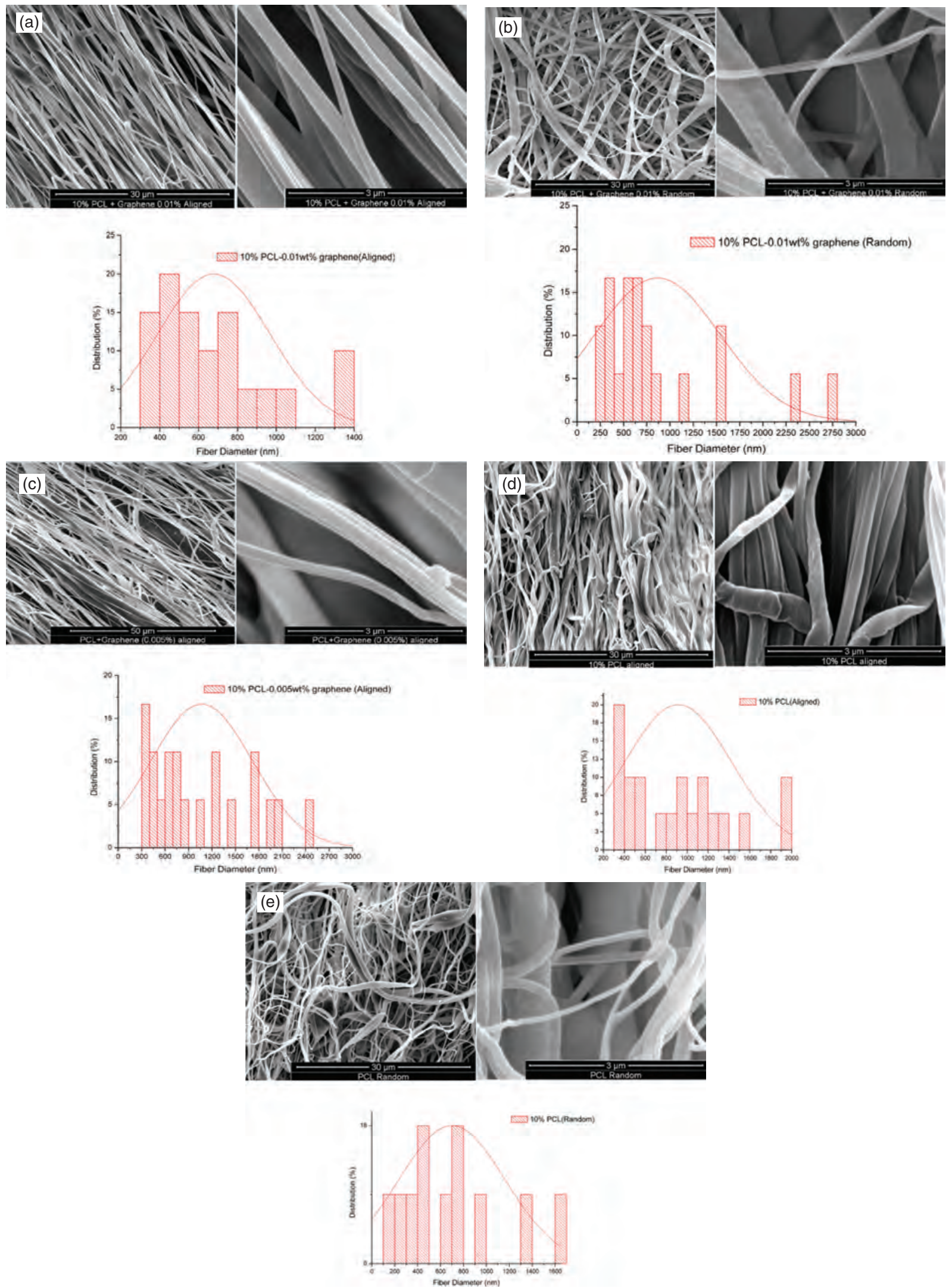

Figure 2. SEM micrograph of various samples of 10\% PCL-graphene nanofibers and control 10\% PCL nanofibers along with the histogram of fiber diameter in each case. (a) Aligned 10\% PCL- $0.01 \%$ graphene, (b) random 10\% PCL- $0.01 \%$ graphene, (c) aligned 10\% PCL- $0.005 \%$ graphene, (d) aligned 10\% PCL, (e) random 10\% PCL. 

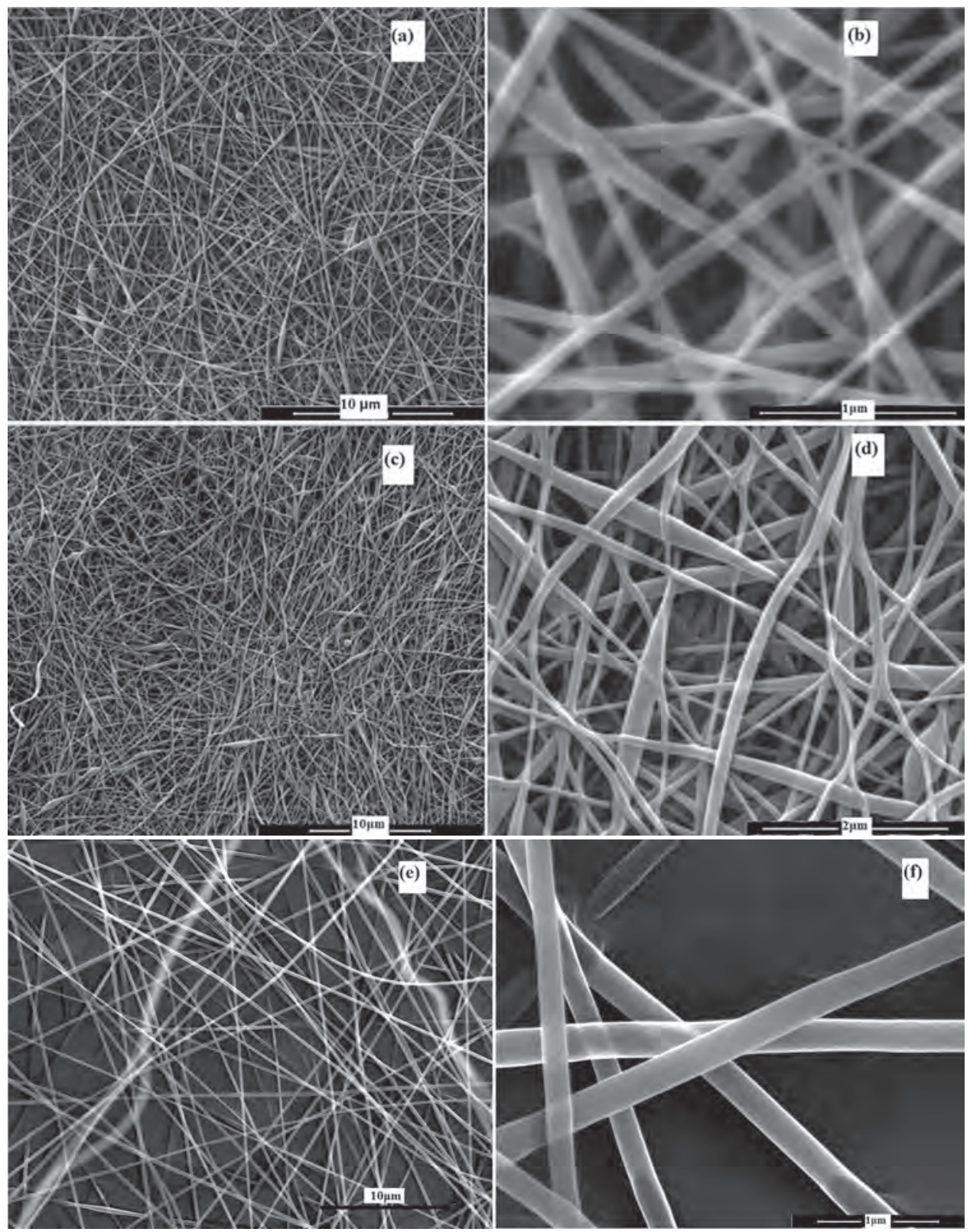

Figure 3. SEM images of randomly oriented PVA composite nanofibers (a), (b) 10\% PVA, (c), (d) 15\% PVA, (e), (f) $10 \%$ PVA-0.1 wt $\%$ MWNTs.

primarily impacted at its periphery by the emanating electric field lines from the needle tip at $15 \mathrm{kV}$ (Figs. 5(A) and (B)). It was realized that the accumulation of nanofibers would be higher at the periphery mainly due to this effect. The resultant plot of electric field $(\mathrm{V} / \mathrm{m})$ with respect to the arc length across the disc in the $y-z$ plane demonstrated that there is a significant difference in the electric field intensity at the periphery compared to the disc $(\sim 10,000 \mathrm{~V} / \mathrm{m})$. The little difference in the electric field between the two peripheral sides of the disc is due to a slight mismatch in the two ends of the arc length across the air box.

\section{MODEL FOR FORMATION OF ALIGNED NANOFIBERS ON THE ROTATING COLLECTOR}

Understanding of the fiber formation from a single drop of polymeric solution is a non-trivial event. The fundamental equations for fiber formation in melt spinning and various strategies used for alignment of nanofibers in the past few years can be used to understand coaxial alignment of fibers using ARC collector. We hypothesize a mathematical model for alignment of nanofibers on ARC collector using the approximations discussed previously ${ }^{28}$ and comparing the theory of fiber alignment on rotating drum collector. ${ }^{21,28}$ As shown in Figure 6, neglecting 

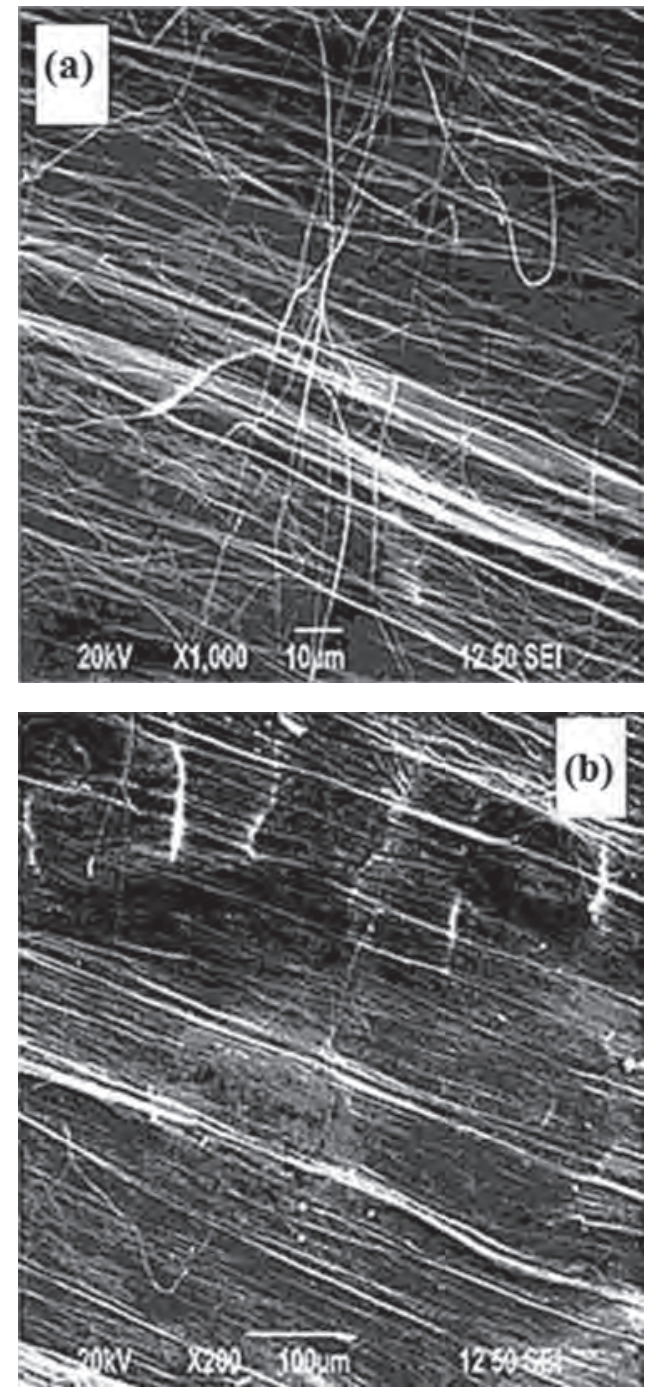

Figure 4. SEM image of aligned $10 \%$ PVA nanofibers using ARC.

gravitational force " $F$ " acting on the drop, the surface tension ' $\sigma$ ' is compensated by force of electric potential across the needle and collector. The droplet is deformed by electric field into conical shape subsequently emerging out as a jet. At this point electric field overcomes the surface tension of the drop.

\subsection{Interaction of Tensile Force Introduced by Rotating Disc}

The tension force produced by the rotating disc collector $\left(F_{\text {rd }}\right)$ and the force due to gravity $\left(F_{\text {gr }}\right)$ are balanced by the rheological force $(\sigma)$ which arises due to the friction between polymer molecules, the aerodynamic force $\left(F_{\mathrm{ar}}\right)$ which is drag exerted by the air flow in the chamber on the fiber jet, the inertial force $\left(F_{\text {ir }}\right)$ and the surface tension of fiber jet $\left(\sigma_{\mathrm{s}}\right) . F_{\text {ir }}$ is a combination of radial forces on charge $(R)$ and cohesive forces among charges $(C)$.

$$
F_{\mathrm{rd}}+F_{\mathrm{gr}}=\sigma+F_{\mathrm{ar}}+F_{\mathrm{ir}}+\sigma_{\mathrm{s}}
$$

Above model is important for stability of fiber jet formation. ${ }^{28}$

Force on a given point on the rotating disc will be centripetal force exerted on that point during rotation in circular path and can be written as,

$$
F_{\text {rd }}=m a_{c}=\frac{m_{t} V_{t}^{2}}{r}
$$

An axial stress generated in the fiber jet is given by equation

$$
\sigma=3 \mu\left(\frac{v_{t}-v_{f}}{l}\right)^{29,30}
$$

Since, fiber jet is subjected to the force of electric field, the surface tension build up in fiber along its cross sectional area will be

$$
\sigma_{\mathrm{s}}=\frac{E}{A}
$$

Gravitational force acting on the fiber jet of mass $m$,

$$
F_{\mathrm{gr}}=m_{f} g
$$

Considering a fiber loops in the median region of setup, Eq. (1) will govern the generation of tensile force in fiber jet and loops. As the fiber loop comes in the nearest region of the collector, the force of rotation of the collector will take up a charge and will generate significant tensile force by the cooperation of electric field strength. Fiber will now deposit starting from its tip, gradually sticking its lateral surface along the circumference of collector. At this point the radius of fiber loop will be stabilized since collector will strongly attract charges within the fiber. Difference between collector velocity $\left(V_{t}\right)$ and fiber velocity $\left(V_{f}\right)$ will gradually decrease and may approximately be zero. We can assume $\mu$ to be constant at the time of deposition. Considering no further elongation at this point we can make area of cross section $(A)$, length $(l)$, and volume $(V)$ of a depositing fiber to be constant. Hence we get

$$
v_{t}^{2}+r \propto E
$$

Equation (6) can be used to explain the phenomenon of normalized fiber deposition as,

(1) The direct proportionality between collector velocity $V_{t}$ and Electric field strength $E$ shows that $V_{t}$ will cooperate with $E$ to add up a force to maintain the constant orientation of fiber.

(2) $r$ indicates that the fiber loop will now keep on depositing along the radius of the collector. In this case loop radius can be treated as a Vector quantity $\bar{r}$, being affected by collector velocity $\left(V_{t}\right)$ and electric field $(E)$ in the space.

(3) The interaction between the electric field and the charge in a polymer jet (fiber) turns the fiber in erratic loops, randomly depositing it on the stationary collector. 

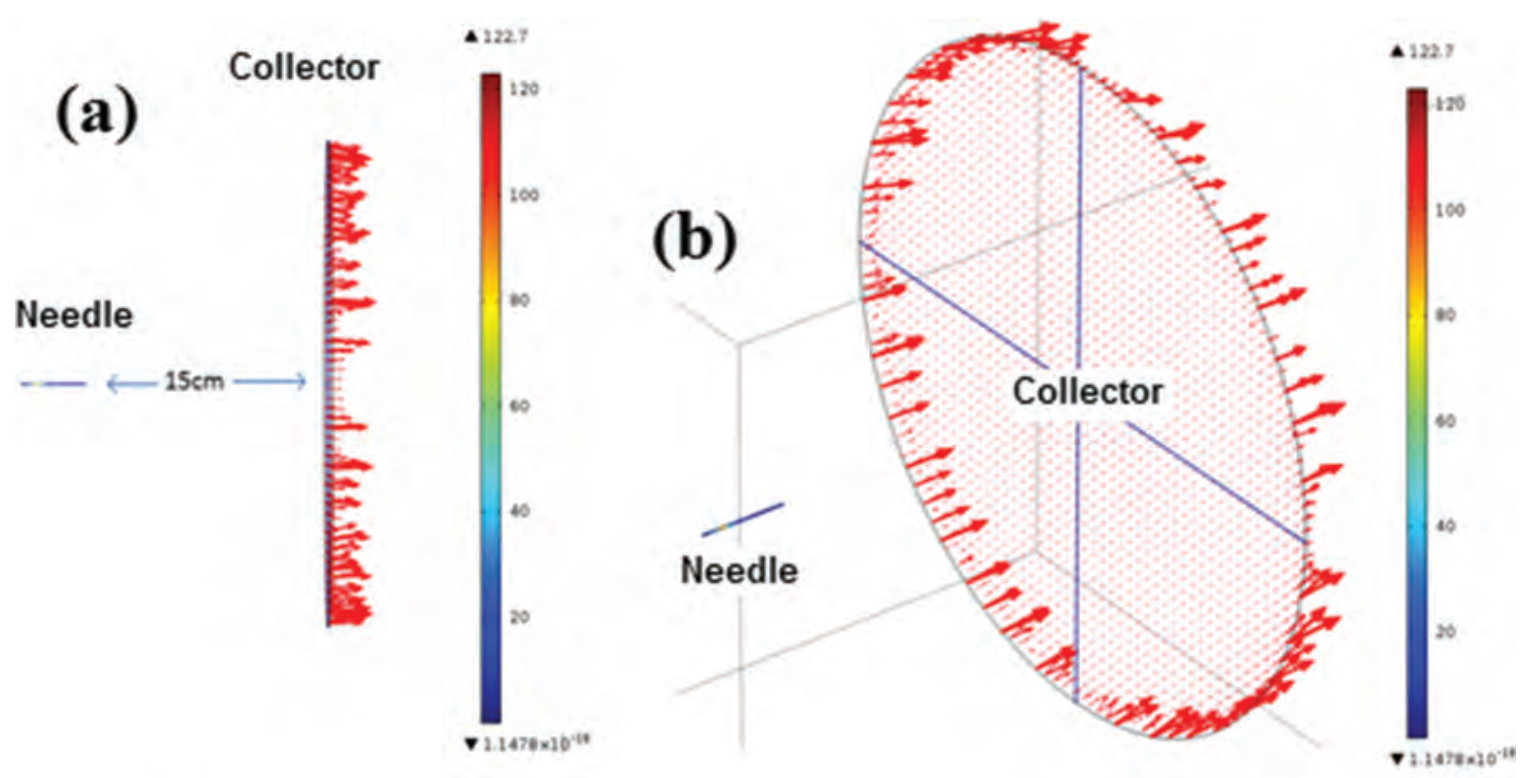

(c)

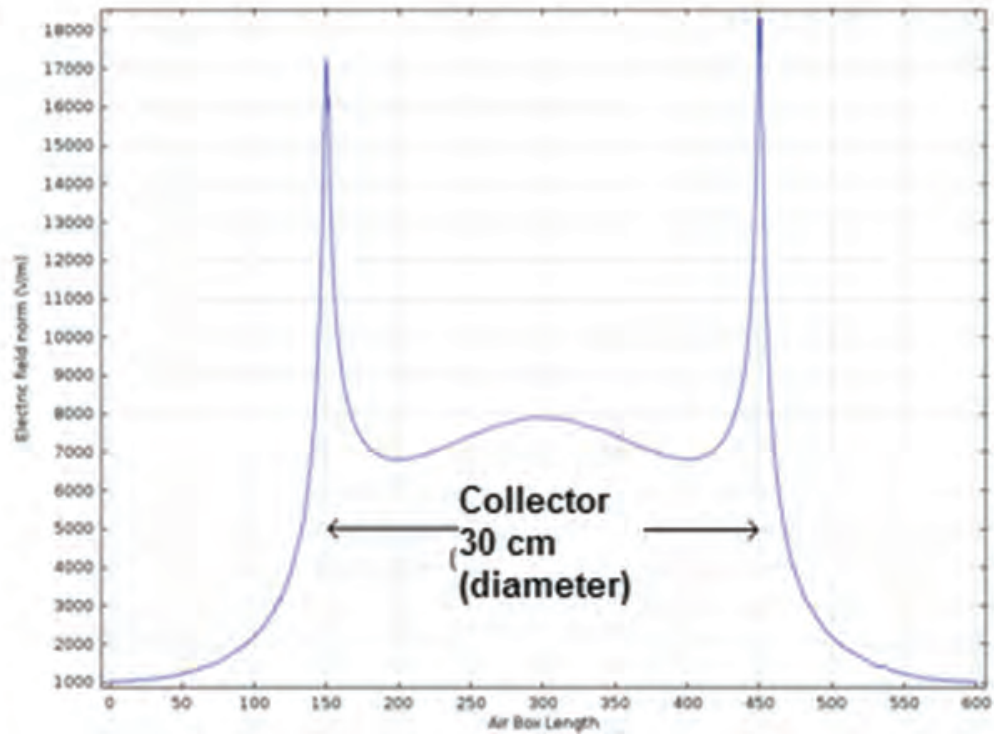

Figure 5. Electric Field modeling and analysis showing the electric field pattern at the collector surface. (a) Distance between the needle (positive terminal) and the collector (ground) showing the electric field lines. The length and thickness of the arrows is proportional to the intensity of the field (maximum of $122.7 \mathrm{~V} / \mathrm{m}$ ), (b) a 3-D view of the model showing the electric field predominantly over the periphery of the disc collector anticipating a larger accumulation of nanofibers over the periphery, (c) line graph plot showing the highest intensity of the electric field at the periphery of the $30 \mathrm{~cm}$ diameter collector. The difference in field strength $(\sim 1000 \mathrm{~V} / \mathrm{m})$ between the two edges is due to the error in positioning the arc across the disc collector.

In order to align fiber coaxially along its circumference, the circular collector should rotate with sufficient rpm high enough and having diameter (radius) large enough to be proportionate to the electric field in the space.

If we consider an electric field $E$ and elongation viscosity $\mu$ to be constant at the point of deposition, radius to be unaffected, and considerable change in fiber velocity then the Eq. (6) will be,

$$
v_{t}^{2} \propto v_{t}-v_{f}
$$

Or

$$
v_{t}^{2}-v_{t} \propto-v_{f}
$$

Or

$$
v_{t}\left(v_{t}-1\right) \propto-v_{f}
$$

Negative sign indicates $v_{f}<v_{t}$.

Above equation explains that collector velocity will be imparted to the fiber velocity and bring it up to the speed 


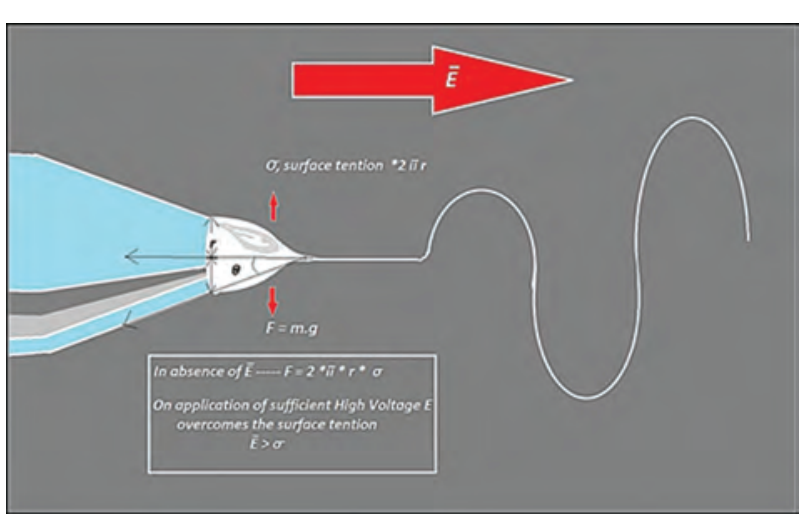

Figure 6. Schematic showing various forces acting on a polymer drop at the tip of needle leading to subsequent stretching.

of collector rotation for normalized deposition. In case of $V_{t}<V_{f}$, the Eq. (7) will be

$$
v_{t}^{2} \propto v_{f}-v_{t}
$$

i.e.,

$$
v_{t}\left(v_{t}+1\right) \propto v_{f}
$$

Equation (11) is still the as Eq. (9) but its positive sign indicates that it is not significantly affected by the collector velocity and therefore factors like elongation viscosity, inertial forces may remain in effect up to appreciable extent. At this point, fiber orientation may vary and wouldn't be depositing itself along the circumference. For instance we will take Eq. (6) in consideration assuming that $\left(v_{t}-v_{f}\right)$ is appreciable quantity as

$$
v_{t}^{2}+r \propto\left(v_{t}-v_{f}\right)+E
$$

Or

$$
v_{t}^{2}+r \propto \Delta V+E
$$

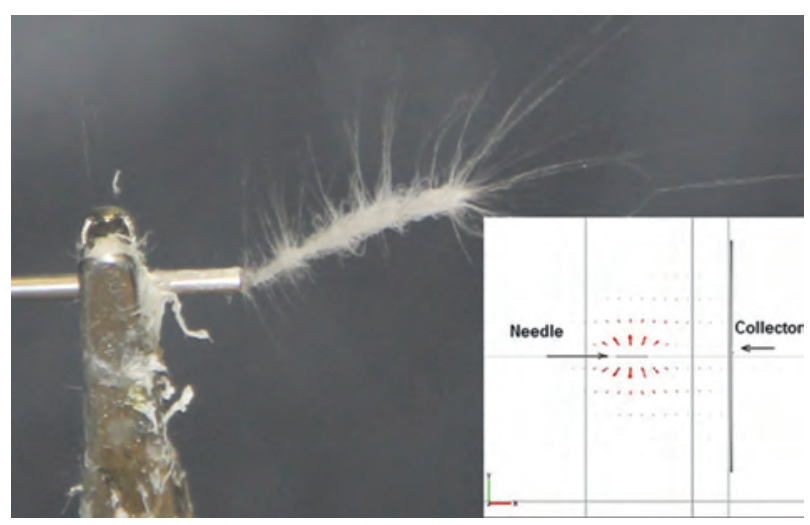

Figure 7. Visualization of the realtime fiber formation from a polymer droplet under electrostatic field and the verification of the electromagnetic field lines between the needle tip and collector surface separated by $15 \mathrm{~cm}$ at $15 \mathrm{kV}$ potential. A close matching with experimental observation and field line simulation is presented here.
Where, $\Delta V=\left(v_{t}-v_{f}\right), v_{t}$ will affect the radius of fiber loop and proportionally maintain fiber velocity $v_{f}$ or $\Delta V$. Because of this over all interaction fiber loop will be acted upon by proportional amount of electric field strength. The entire interaction will result in the phenomenon of normalized deposition.

\section{CONCLUSION}

The capabilities of ARC to produce aligned hybrid nanofibers have been experimentally demonstrated and mathematically modeled. It is observed that the presence of graphene and MWNTs in the PCL and PVA solution respectively, influences the geometry of hybrid nanofibers. Electric field simulation using finite element analysis demonstrated that the field largely develops at the periphery of the collector surface. Mathematical models presented here explain the formation and alignment of nanofibers. Further, it is demonstrated experimentally that the presence of conductive component such as graphene and MWNTs help electrical charges to concentrate on the beads that act as electrical stress centers which possibly lead to continuous stretching of fibers.

\section{ABBREVIATIONS}

ARC Axially Rotating Collector,

MWCNT Multiwall Carbon Nanotubes, PVA Polyvinyl Alcohol, PCL Polycaprolactone.

Acknowledgment: We would like to thank Mr. Jeff Gilarde of Wesleyan University for his assistance performing scanning electron microscopy.

\section{References and Notes}

1. D. Li and Y. Xia, Adv. Mater. 16, 1151 (2004).

2. G. Srinivasan and D. H. Reneker, Polym. Int. 36, 195 (1995).

3. D. Li, Y. Wang, and Y. Xia, Nano Lett. 3, 1167 (2003).

4. A. Frenot and I. S. Chronakis, Curr. Opin. Colloid Interface Sci. 8,64 (2003).

5. L. S. Nair and C. T. Laurencin, J. Bone Joint Surg. Am. 90, 128 (2008).

6. K. Athanasiou, G. Niederauer, and C. Agrawal, Biomaterials 17, 93 (1996).

7. D. Il Cha, H. Y. Kim, K. H. Lee, Y. C. Jung, J. W. Cho, and B. C. Chun, J. Appl. Polym. Sci. 96, 460 (2005).

8. X.-S. Wang, H.-S. Fu, and G.-D. Li, Recent Pat. Nanotechnol. 3, 21 (2009).

9. S. Aryal, C. K. Kim, K.-W. Kim, M. S. Khil, and H. Y. Kim, Mater. Sci. Eng. C 28, 75 (2008).

10. H. Matsumoto, S. Imaizumi, Y. Konosu, M. Ashizawa, M. Minagawa, A. Tanioka, W. Lu, and J. M. Tour, ACS Appl. Mater. Interfaces 5, 6225 (2013).

11. A. H. C. Neto, N. M. R. Peres, K. S. Novoselov, and A. K. Geim, Rev. Mod. Phys. 81, 109 (2009).

12. D. Li, M. B. Müller, S. Gilje, R. B. Kaner, and G. G. Wallace, Nat. Nanotechnol. 3, 101 (2008).

13. R. H. Baughman, A. A. Zakhidov, and W. A. de Heer, Science 297, 787 (2002). 
14. S. Stankovich, D. A. Dikin, G. H. B. Dommett, K. M. Kohlhaas, E. J. Zimney, E. A. Stach, R. D. Piner, S. T. Nguyen, et al., Nature 442, 282 (2006).

15. M. R. Modesti, S. Sundarrajan, D. P. Michele, and S. Ramakrishna, Nanotechnology 19, 285707 (2008).

16. S. G. K., R. J., S. P. N., and C. T. Laurencin, Biomed. Mater. 3, 34002 (2008).

17. W. J. Li, Y. J. Jiang, and R. S. Tuan, Tissue Eng. 12,1775 (2006).

18. D. Kai, G. Jin, M. P. Prabhakaran, and S. Ramakrishna, Biotechnol. J. 8, 59 (2013).

19. J. Doshi and D. H. Reneker, J. Electrostat. 35, 151 (1995).

20. W. E. T. and S. Ramakrishna, Nanotechnology 16, 1878 (2005).

21. P. Katta, M. Alessandro, R. D. Ramsier, and G. G. Chase, Nano Lett. 4, 2215 (2004).

22. J. P. Berry, Electrostatically Produced Structures and Methods of Manufacturing, US Patent 4965110 (1990).
23. A. Bornat, Production of electrostatically spun products, US Patent 4689186 (1987)

24. A. Theron, E. Zussman, and A. L. Yarin, Nanotechnology 12, 384 (2001).

25. R. Dersch, T. Liu, A. K. Schaper, A. Greiner, and J. H. Wendorff, J. Polym. Sci. Part A Polym. Chem. 41, 545 (2003).

26. J. M. Deitzel, J. D. Kleinmeyer, J. K. Hirvonen, and N. C. B. Tan, Polymer (Guildf.) 42, 8163 (2001).

27. S. Chuangchote and P. Supaphol, J. Nanosci. Nanotechnol. 6, 125 (2006).

28. A. Ziabicki, Fundamentals of Fibre Formation? The Science of Fibre Spinning and Drawing, Wiley, London, New York (1976).

29. M. Lewin, Handbook of Fiber Chemistry, Third edn., CRC, New York (2006).

30. G. E. Hagler, Polym. Eng. Sci. 21, 121 (1981).

Received: 17 November 2014. Accepted: 12 January 2015. 